\title{
Video Article \\ Study of Viral Vectors in a Three-dimensional Liver Model Repopulated with the Human Hepatocellular Carcinoma Cell Line HepG2
}

\author{
Thomas Hiller ${ }^{1}$, Viola Röhrs ${ }^{1}$, Eva-Maria Dehne ${ }^{2}$, Anke Wagner ${ }^{1,3}$, Henry Fechner ${ }^{1}$, Roland Lauster ${ }^{2}$, Jens Kurreck ${ }^{1}$ \\ ${ }^{1}$ Department of Applied Biochemistry, Institute of Biotechnology, Berlin University of Technology \\ ${ }^{2}$ Department of Medical Biotechnology, Institute of Biotechnology, Berlin University of Technology \\ ${ }^{3}$ Department of Bioprocess Engineering, Institute of Biotechnology, Berlin University of Technology
}

Correspondence to: Jens Kurreck at jens.kurreck@tu-berlin.de

URL: https://www.jove.com/video/54633

DOI: doi:10.3791/54633

Keywords: Cancer Research, Issue 116, adeno-associated virus vectors, extracellular matrix, liver, recellularization, RNA interference, short hairpin RNA, bioengineering

Date Published: $10 / 24 / 2016$

Citation: Hiller, T., Röhrs, V., Dehne, E.M., Wagner, A., Fechner, H., Lauster, R., Kurreck, J. Study of Viral Vectors in a Three-dimensional Liver Model Repopulated with the Human Hepatocellular Carcinoma Cell Line HepG2. J. Vis. Exp. (116), e54633, doi:10.3791/54633 (2016).

\section{Abstract}

This protocol describes the generation of a three-dimensional (3D) ex vivo liver model and its application to the study and development of viral vector systems. The model is obtained by repopulating the extracellular matrix of a decellularized rat liver with a human hepatocyte cell line. The model permits studies in a vascularized 3D cell system, replacing potentially harmful experiments with living animals. Another advantage is the humanized nature of the model, which is closer to human physiology than animal models.

In this study, we demonstrate the transduction of this liver model with a viral vector derived from adeno-associated viruses (AAV vector). The perfusion circuit that supplies the 3D liver model with media provides an easy means to apply the vector. The system permits monitoring of the major metabolic parameters of the liver. For final analysis, tissue samples can be taken to determine the extent of recellularization by histological techniques. Distribution of the virus vector and expression of the delivered transgene can be analyzed by quantitative PCR (qPCR), Western blotting and immunohistochemistry. Numerous applications of the vector model in basic research and in the development of gene therapeutic applications can be envisioned, including the development of novel antiviral therapeutics, cancer research, and the study of viral vectors and their potential side effects.

\section{Video Link}

The video component of this article can be found at https://www.jove.com/video/54633/

\section{Introduction}

Most current biomedical research relies on one of two approaches, either two-dimensional (2D) cell culture experiments or animal models, which are three-dimensional (3D) by their very nature. However, these approaches have some severe drawbacks. Cells grown in 2D culture have been shown to differ in gene expression patterns and cell physiology from those cultivated under 3D conditions. ${ }^{1}$ Animal models, in addition to being associated with ethical concerns, often do not model human physiology well. Although the lack of obvious toxic effects of a compound must be confirmed in animal models prior to the first dosing in humans, multiple cases have been documented in which severe, sometimes fatal, adverse effects have occurred in clinical trials. ${ }^{2}$

To overcome these shortcomings, humanized 3D ex vivo organ models have become important research tools. When cultivated under suitable conditions, cells self-assemble into 3D structures known as spheroids. However, these spheroids lack a vascular system, which limits the distribution of small molecular compounds, large biologics and viral vectors alike. For example, adenoviral vectors only transduced the outer cell layers of spheroids prepared from human glioblastomas. ${ }^{3} \mathrm{~A}$ solution to this problem is the use of an organ model containing a vascular system. To this end, the organ of interest can be explanted from an animal, and the animal cells can be replaced by human cells. Various methods for decellularization of animal livers by treatment with detergents or sodium cholate have been described. ${ }^{4-6}$ The resulting extracellular matrix (ECM) harbors cytokines and growth factors which regulate various cellular processes. ${ }^{7}$ It can be used as a scaffold for recellularization with human cells to obtain a functional organ model.

In a recent study, we used a humanized 3D liver model to study distribution and transgene expression of an adeno-associated virus (AAV) vector. ${ }^{8} \mathrm{AAV}$ vectors belong to the most promising viral vectors for gene therapeutic applications. ${ }^{9}$ The first, and to date only, approved gene therapeutic intervention in the Western world uses an AAV vector for the transfer of lipoprotein lipase. ${ }^{10}$ 


\section{Protocol}

NOTE: R.L. obtained ethical approval for the explantation of organs from the Landesamt für Gesundheit und Soziales (LaGeSo). Livers were explanted from Wister rats. The inferior vena cava and the portal vein of the liver were cannulated with a $22 \mathrm{G}$ cannula. Methods for the decellularization of explanted livers have been described previously. ${ }^{4,5}$ The extracellular matrices used here were obtained by excessive perfusion of a rat liver with $1 \%$ sodium deoxycholate.

\section{Recellularization of Extracellular Matrix (ECM) of a Rat Liver}

1. Expansion of hepatic cell line HepG2

1. Culture the hepatocellular carcinoma cell line HepG2 in Roswell Park Memorial Institute (RPMI) 1640 medium supplemented with $10 \%$ fetal calf serum, $2 \mathrm{mM}$ glutamine, and $2 \mathrm{mM}$ of penicillin and streptomycin, each.

2. Seed $1.5 \times 10^{7}$ cells in T175 bottles and grow the cells at $37^{\circ} \mathrm{C}$ and $5 \% \mathrm{CO}_{2}$. Harvest cells after 4 days by trypsinization for 5 min at 37 ${ }^{\circ} \mathrm{C}, 5 \% \mathrm{CO}_{2}$.

3. Spin down the cells at $300 \times \mathrm{g}$ and resuspend them in $4 \mathrm{ml} \mathrm{PBS}$ and count the cells with a Neubauer chamber under a microscope. One T175 bottle will yield approximately $4.5 \times 10^{7}$ cells.

NOTE: Ensure the culture contains sufficient cell numbers for recellularization of the rat liver ECM with $6 \times 10^{8} \mathrm{HepG} 2 \mathrm{cells}$ per ECM $\left(10 \times 175 \mathrm{~cm}^{2}\right.$ culture flasks with $4-5 \times 10^{8}$ cells each).

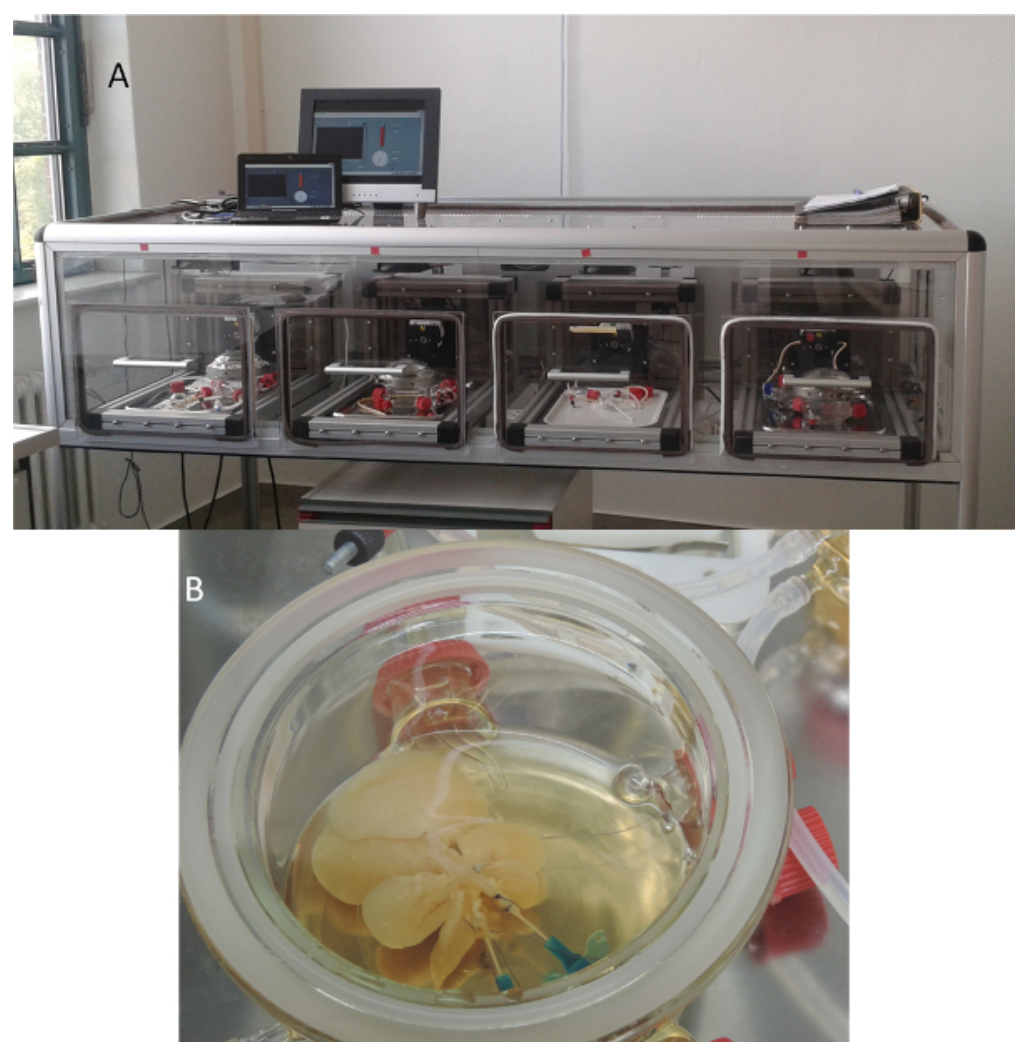

Figure 1. Bioreactor system. A) This bioreactor system was custom-made. It maintains the organ models at $37{ }^{\circ} \mathrm{C}$ and $5 \% \mathrm{CO}_{2}$. The flow rates of the peristaltic pumps can be regulated individually. B) The liver is placed in a growth chamber and connected to the perfusion circuit, which consists of a peristaltic pump, a medium reservoir, a bubble trap and a pressure sensor. Please click here to view a larger version of this figure.

2. Recellularization of the ECM

1. Set up the bioreactor system, containing a liver perfusion chamber, a perfusion system, a reservoir of medium and a bubble trap. Sterilize the bioreactor system $\left(121^{\circ} \mathrm{C}, 15 \mathrm{~min}\right)$.

2. Connect the bioreactor system with a peristaltic pump and place it in an incubator providing appropriate conditions $\left(37{ }^{\circ} \mathrm{C}, 5 \% \mathrm{CO}_{2}\right)$. Place decellularized rat liver scaffolds ${ }^{8}$ in the liver perfusion chamber of the bioreactor system (Figure 1).

3. Connect the cannulated portal vein and vena cava using tube clips to the perfusion system. Equilibrate scaffold with $150 \mathrm{ml}$ RPMI medium (as described in 1.1) over $5 \mathrm{~d}$ with a flow rate of $1.25 \mathrm{ml} / \mathrm{min}$.

4. Disconnect the liver scaffold from the media circuit and inoculate the scaffold with $3 \times 10^{8} \mathrm{HepG} 2$ cells (in $5 \mathrm{ml}$ ) via the portal vein using a $5 \mathrm{ml}$ syringe, avoid air bubble formation and allow the cells to repopulate the ECM by incubating them for $1 \mathrm{hr}$ in the scaffold with the pump switched off.

5. Gradually increase the flow rate by adjusting the pump, starting with $1.25 \mathrm{ml} / \mathrm{min}$ for $10 \mathrm{~min} ; 2.5 \mathrm{ml} / \mathrm{min}$ for $20 \mathrm{~min}$ and finally $3.75 \mathrm{ml} /$ $\min$ for $30 \mathrm{~min}$.

6. Repeat steps 1.2.4-1.2.5 to reach a total cell number of $6 \times 10^{8}$. 
7. Run the bioreactor system at a flow rate of $3.75 \mathrm{ml} / \mathrm{min}$. Culture the recellularized rat liver for 2 weeks.

NOTE: Replace one third of the medium with fresh medium $(50 \mathrm{ml})$ every other day. Sample the culture medium to measure physiological parameters, such as lactate dehydrogenase activity, the $\mathrm{pH}$ of medium samples and the concentration of glucose and lactate.

\section{Transduction of the Recellularized Rat Liver}

1. Large-Scale Production of AAV Vectors

1. Produce, purify, and quantify AAV vectors as described previously: ${ }^{11}$

1. Briefly, produce AAV vectors in roller bottles and purify them by iodixanol gradient centrifugation. Remove residual iodixanol by filtration over PD10 gel filtration columns. Determine the AAV vector concentration by qPCR using genomic AAV DNA as a standard.

NOTE: The self-complementary, pseudotyped AAV2/6 vector used in the present study encoded EmGFP as a reporter to demonstrate transduction efficiency and an shRNA expression cassette for the knockdown of an endogenously expressed gene (human cyclophilin $B(h C y c B)$, Figure 2). Ensure a sufficient amount of pseudotyped scAAV vectors of serotype $6\left(2.7 \times 10^{13}\right.$ AAV vectors per liver model).

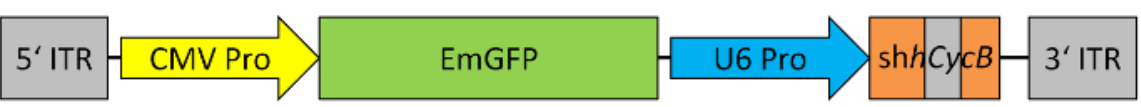

Figure 2. Map of the self-complementary, pseudotyped AAV2/6 vector used in the present study. The genome consists of the inverted terminal repeats (ITR) of AAV2 and encodes EmGFP under control of the CMV promoter as well as an shRNA under control of a U6 promoter. Please click here to view a larger version of this figure.

2. Transduction of Liver Model

1. Adjust the AAV vector solution to a final concentration of $2.7 \times 10^{13}$ vector genomes in $5 \mathrm{ml}$ by adding the respective volume of PBS

2. Disconnect the liver from the media circuit by removing the tubing from the cannula. Connect a $5 \mathrm{ml}$ syringe to the cannula of the portal vein and inject the complete AAV vector solution $(5 \mathrm{ml})$. NOTE: Optionally, add phenol red $(5 \mu \mathrm{g} / \mathrm{ml})$ to follow the distribution of the AAV vector solution throughout the liver.

3. Incubate for $1 \mathrm{hr}$ without pumping. Gradually increase flow rate, starting with $1.25 \mathrm{ml} / \mathrm{min}$ for $10 \mathrm{~min} ; 2.5 \mathrm{ml} / \mathrm{min}$ for $20 \mathrm{~min}$ and $3.75 \mathrm{ml} /$ $\min$ for $30 \mathrm{~min}$. Culture the recellularized rat liver over 6 days.

NOTE: Replace one third of the medium as given in NOTE under 1.2.7

\section{Assessment of the Recellularized Transduced Rat Liver}

1. Hematoxylin and eosin (HE) staining and Immunohistochemical Analysis

1. Take samples $(0.5 \times 0.5 \times 1.5-2 \mathrm{~cm})$ from each liver lobe using a scalpel.

2. Incubate samples (from 3.1.1) in $4 \%$ paraformaldehyde (PFA) $+4 \%$ sucrose solution for $1.5 \mathrm{hr}$ at $4{ }^{\circ} \mathrm{C}$. (Caution: PFA is toxic and carcinogenic. Always keep PFA within a fume hood and wear appropriate protective clothing.) Wash three times with PBS (1 min per wash step) and incubate in $8 \%$ sucrose overnight at $4{ }^{\circ} \mathrm{C}$.

3. Pour fixing medium into plastic cryomolds, place the samples into fixing medium free of air bubbles. Add fixing medium until sample is well covered. Store embedded samples at $-80^{\circ} \mathrm{C}$ until further use.

4. Prepare cryo-sections $(10 \mu \mathrm{m})$ with a cryotome ${ }^{12}$. Assess recellularization by hematoxylin + eosin staining ${ }^{8}$. Assess transduction efficiency by immunohistochemical staining for the gene-of-interest (here: EmGFP).

2. Molecular biological sampling

1. Sample each liver lobe with a biopsy punch (4 mm in diameter). Isolate total RNA, DNA and proteins according to the manufacturer's instructions for the assessment of transgene expression of EmGFP, and $h C y c B$ knock down ${ }^{8}$.

\section{Representative Results}

For the assessment of the extent of recellularization, cryo-sections were prepared from each lobe of each recellularized liver model. The sections were then analyzed by hematoxylin and eosin staining. As can be seen in Figure 3, each lobe of the three liver models, denoted as TLM1, TLM2 (transduced liver models $1+2$ ) and Ctrl (control liver model that was recellularized, but not transduced), were repopulated with HepG2 cells. This hepatocellular carcinoma cell line was established from the tumor tissue of a 15-year-old Argentine boy. Some areas of the liver models were more intensively recellularized than others. The reasons for these variations remain to be determined. 


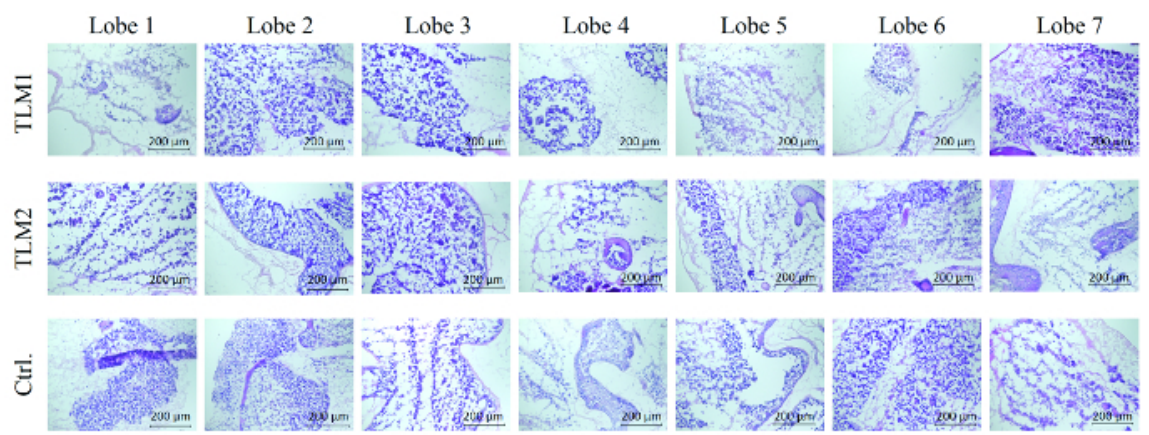

Figure 3. Hematoxylin and eosin stain of each lobe of the three analyzed liver models. TLM1+2: transduced liver models 1 and 2; Ctrl.: control liver model that was recellularized but not transduced. Scale bar: $200 \mu \mathrm{m}$. (Re-printed with permission from Ref. 8.) Please click here to view a larger version of this figure.

In the next step, the transduction of the liver models was assessed. To this end, DNA was prepared from 28 punch biopsies of each of the liver models and the vector titer was quantified by qPCR. On average, 55 and 90 internalized vector genomes per cell (VG/cell) were measured for the two transduced liver models. Experiments in 2D cell culture have shown $30 \mathrm{VG} / \mathrm{cell}$ are sufficient for strong transgene expression and RNAimediated silencing. Production of EmGFP was analyzed by RT-PCR and Western blotting. In fact, expression of the reporter was detected in $80-90 \%$ of the biopsies on the mRNA and protein levels, respectively. ${ }^{8}$ To obtain a comprehensive picture of the transduction efficiency, cryosections were immunohistologically analyzed. As can be seen in Figure 4, areas of the liver model that were successfully recellularized, as visualized by DAPI staining, also gave strong fluorescent signals in the immunohistochemical analysis of EmGFP expression. As expected, the control liver model, not treated with AAV vectors, did not show any EmGFP expression.

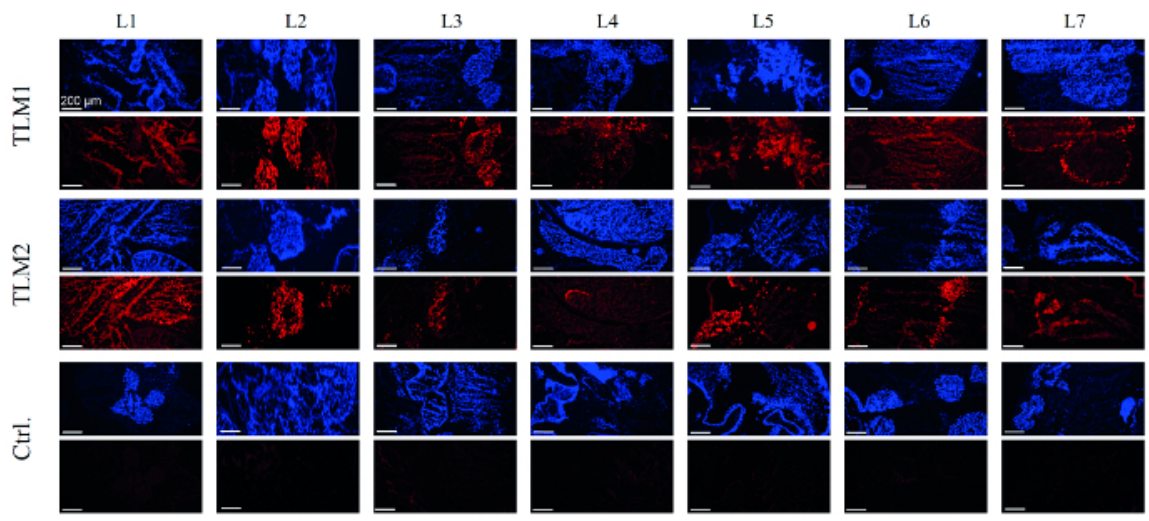

Figure 4. Immunohistochemical analysis of EmGFP expression. Cells that repopulated the liver models were visualized by DAPI staining (blue); EmGFP expression was visualized by immunochemical staining (red). TLM1+2: transduced liver models 1 and 2; Ctrl.: control liver model that was recellularized but not transduced. Scale bar: $200 \mu \mathrm{m}$. (Re-printed with permission from Ref. 8.) Please click here to view a larger version of this figure.

In a final test, AAV-mediated knockdown of $h C y c B$ was analyzed by qRT-PCR. The knockdown of $h C y c B$ was found to be between 70 and $90 \%$, averaged over all lobes of the two transduced liver models (Figure 5). 


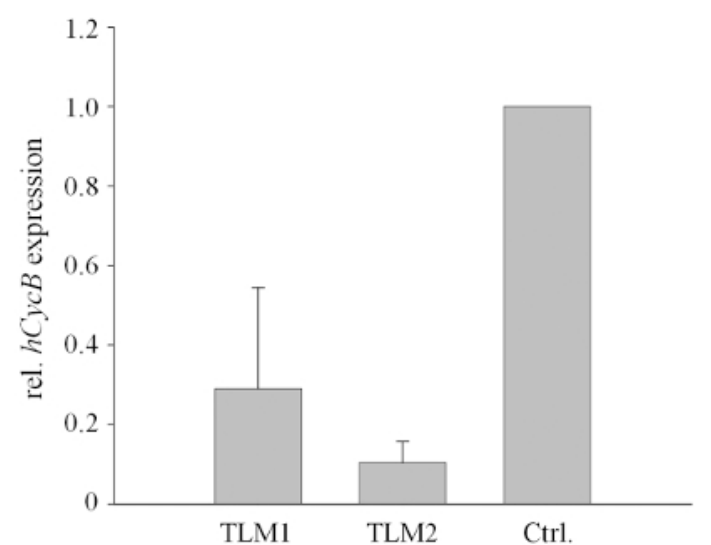

Figure 5. RNAi-mediated knockdown of $h C y c B$ in AAV-transduced 3D liver models. Silencing of $h C y c B$ was determined by qRT-PCR. Mean values and standard deviations (SD) were calculated for all samples of each transduced 3D liver model (TLM1 and 2, respectively) and normalized to the mean value of the non-transduced control (Ctrl.). (Re-printed with permission from Ref. 8.) Please click here to view a larger version of this figure.

\section{Discussion}

The reconstituted 3D livers described here provide a model to study viral vectors in a humanized system. Repopulation of the ECM of a rat liver with a human hepatocellular carcinoma cell line generates a vascularized system which permits the study of large biologics. These results provide a proof-of-concept that the reconstituted liver model can be efficiently transduced with a viral vector.

For the experiments shown here, each and every lobe of the liver was transduced by the AAV vector. However, in some preliminary experiments, single lobes were not repopulated with cells. It is therefore important to prevent cell debris or other components from occluding the vascular system. To test whether all lobes can be perfused, a non-toxic dye such as phenol red can be flushed through the liver model.

Another crucial matter is keeping the liver scaffold sterile. While treatment with ethanol or antibiotics is disadvantageous for the vascular system, irradiation of the extracellular matrix with $y$-radiation preserved the vessels and sterilized the sample.

Furthermore, the size of explanted livers will differ, so that the number of cells used for the recellularization procedure and the repopulation time may have to be adjusted to obtain reproducible results.

The rat livers used in the present study are comparatively large and require large amounts of cells and test reagents (e.g., AAV vectors). In addition, the repopulation procedure took more than two weeks. This limits the number of replicates which can be done with reasonable effort We are currently establishing the model for mouse livers, which are only approximately one fifth of the volume of rat livers, allowing the use of fewer cells and less test reagents. Although proportional scale-down of the cell number seems reasonable, the exact amounts need to be determined in further experiments.

Another shortcoming of the present model is the use of the hepatocellular HepG2 cell line. Experiments are ongoing to develop the use of hepatocytes differentiated from induced pluripotent stem cells, which will provide a physiologically more relevant model. Furthermore, the liver consists of multiple cell types in addition to the hepatocytes, e.g., Kupffer cells and sinosoids. We assume that the different cell types will repopulate their natural environments when an ECM is recellularized with multiple cell types.

The 3D liver model combines several advantages. A major disadvantage of conventional in vivo models is that animal physiology differs substantially from human physiology. Toxic side effects of the treatment of a human patient may therefore remain undetected. This shortcoming can be overcome by reconstituting the 3D liver model with human cells that more closely reflect the biology of human patients.

The second advantage of the liver model is its contribution to animal welfare. Although animal components are required for the reconstitution experiments, the approach still follows the aims of the $3 \mathrm{R}$ principle (replacement, reduction, refinement), as surplus animals can be used that were sacrificed for other animal experiments, i.e., no additional animals are needed and the approach completely avoids suffering of animals which is frequently associated with in vivo experiments. Spheroids are an alternative tool to study cellular processes in a 3D system. However, spheroids are not vascularized so that large substances and biologics do not penetrate deeply into the inner parts of the structure. These problems have been overcome with the vascularized 3D liver model.

In the experiments described here, AAV vectors were investigated, since they are among the most promising candidates for gene therapeutic applications. As numerous gene therapeutic approaches aim at targeting the liver, e.g., for the treatment of infections with hepatitis viruses or of alpha-1-antitrypsin deficiency, the 3D liver can be used in the process of these AAV vector development. It is, of course, also suitable for the study of other hepatotropic viral vectors, e.g., adenoviral vectors. In addition, it can be used to study infectious hepatitis viruses such as hepatitis $\mathrm{B}$ or $\mathrm{C}$ virus. It can, for example, be employed to design new antiviral strategies. Moreover, 3D organ models represent promising tools to develop new cytostatic therapeutics to treat cancer and to carry out toxicological studies. On the long run, artificial livers may be used in regenerative medicine as transplants. Taken together, the 3D liver model offers a wide range of applications in infection biology and other fields of biomedical research. 


\section{Disclosures}

The authors have nothing to disclose.

\section{Acknowledgements}

The authors thank Bernd Krostitz for technical assistance, Radoslaw Kedzierski for initial contributions to the project, Erik Wade for proofreading and giving helpful comments, and Prof. Heike Walles for providing the bioreactor and sharing her valuable experience with organ decellularization. We are also thankful for funding of the project and publication by the Berlin University of Technology.

\section{References}

1. Chang, T.T., Hughes-Fulford, M. Monolayer and spheroid culture of human liver hepatocellular carcinoma cell line cells demonstrate distinct global gene expression patterns and functional phenotypes. Tissue Eng. Part A. 15 (3), 559-567 (2009).

2. Butler, D., Callaway, E. Scientists in the dark after French clinical trial proves fatal. Nature. 529 (7586), 263-264 (2016).

3. Enger, P.O., Thorsen, F., Lonning, P.E., Bjerkvig, R., Hoover, F. Adeno-associated viral vectors penetrate human solid tumor tissue in vivo more effectively than adenoviral vectors. Hum. Gene Ther. 13 (9), 1115-1125 (2002).

4. Uygun, B.E. et al. Decellularization and recellularization of whole livers. J. Vis. Exp. (48), e2394 (2011).

5. Hillebrandt, K. et al. Procedure for Decellularization of Rat Livers in an Oscillating-pressure Perfusion Device. J. Vis. Exp. (102), e53029 (2015).

6. Baptista, P.M. et al. The use of whole organ decellularization for the generation of a vascularized liver organoid. Hepatology. 53 (2), $604-617$ (2011).

7. Yagi, H. et al. Human-scale whole-organ bioengineering for liver transplantation: a regenerative medicine approach. Cell Transplant. 22 (2), 231-242 (2013).

8. Wagner, A. et al. Use of a three-dimensional humanized liver model for the study of viral gene vectors. J. Biotechnol. 212 134-143 (2015).

9. Kay, M.A. State-of-the-art gene-based therapies: the road ahead. Nat. Rev. Genet. 12 (5), 316-328 (2011).

10. Yla-Herttuala, S. Endgame: glybera finally recommended for approval as the first gene therapy drug in the European union. Mol. Ther. 20 (10), 1831-1832 (2012).

11. Wagner, A., Röhrs, V., Kedzierski, R., Fechner, H., Kurreck, J. A novel method for the quantification of adeno-associated virus vectors for RNA interference applications using quantitative polymerase chain reaction and purified genomic adeno-associated virus DNA as a standard. Hum. Gene Ther. Methods. 24 (6), 355-363 (2013).

12. Takagi, H. et al. Microdissected region-specific gene expression analysis with methacarn-fixed, paraffin-embedded tissues by real-time RTPCR. J. Histochem. Cytochem. 52 (7), 903-913 (2004). 